\title{
A UTILIZAÇÃO E PRODUÇÃO DIDÁTICA DE BIBLIOTECAS DIGITAIS NO PROCESSO DE DETALHAMENTO DO PROJETO ARQUITETÔNICO
}

\author{
THE UTILIZATION AND DIDATIC PRODUCTION OF DIGITAL LIBRARIES IN \\ DETAILING PROCESS OF ARCHITECTURAL DESIGN
}

\author{
Etiene do Amaral ARCARI \\ Arquiteta mestranda em Arquitetura e Urbanismo, POSARQ - UFSC, Centro Tecnológico (CTC), etiene.a@posgrad.ufsc.br.
}

\begin{abstract}
This article discusses how some digital technologies applied to the design process can contribute to the development of architectural detail, serving as a tool for assessment of master research project which has the theme " The Utilization And Didatic Production Of Digital Libraries In Detailing Process Of Architectural Design". The research intends to establish a routine for developing architectural details that explore digital technologies and assist in the teaching and learning project through student experiments process as well as provide a digital library of parametric models of detail amenable to prototyping.
\end{abstract}

Keywords: Digital Technologies, Design Process, Architectural Detailing.

\begin{abstract}
Resumo
O presente artigo objetiva discutir como algumas tecnologias digitais aplicadas ao processo de projeto podem contribuir para o desenvolvimento de detalhes arquitetônicos, servindo como caso de aplicação do projeto de pesquisa de mestrado que tem como tema "A Utilização e Produção Didática de Bibliotecas Digitais no Processo de Detalhamento do Projeto Arquitetônico". A pesquisa tenciona criar uma rotina para desenvolvimento de detalhes arquitetônicos através de experiências didáticas, utilizando tecnologias digitais que auxiliem no processo de ensino e aprendizagem de projeto, assim como disponibilizar uma biblioteca digital com modelos paramétricos de detalhamento passíveis de prototipagem.
\end{abstract}

Palavras-chave :Tecnologias Digitais, Processo de Projeto, Detalhamento Arquitetônico.

\section{Introdução}

A concepção do projeto arquitetônico se relaciona diretamente com a organização de ideias, estudos e métodos que resultarão na representação deste conjunto de elementos, tanto teóricos quanto práticos.

Dentro do desenvolvimento do processo projetual, a concepção da arquitetura atravessa diferentes estágios desde a idealização até a representação técnica que viabiliza a construção do objeto (detalhamento executivo). Apesar da importância da representação destes detalhes, o ensino acadêmico muitas vezes não atinge o nível de explanação necessária para que o projeto complete sua trajetória e então se torne um objeto concebido.
FORD ressalta em seu livro a importância destes detalhes, levando em conta que mesmo sendo fundamentais, pertencem também a um contexto que deve ser considerado:

"Detalhes são a base para - não um acessório para - a compreensão de um edifício. Isto não é para dizer que o detalhe contém em si a ideia da totalidade do edifício; [...] uma compreensão da construção não pode ser separada de uma compreensão do detalhe [...] ". (FORD , 2011, p 13)

Já Marco Frascari acredita que é necessária a compreensão e valorização de cada detalhe arquitetônico, bem como sua representação gráfica. $\mathrm{O}$ resultado é um processo projetual mais claro, definido e maior 
compreensão do campo visual, considerando que as possibilidades de inovação e invenção estão nos detalhes.

FRASCARI vê a arte do detalhamento na união entre materiais, elementos, componentes e partes de determinada construção, de maneira funcional e estética. Acredita que neste contexto existe uma complexidade tão grande, que um mesmo detalhe pode se adequar perfeitamente num edifício, mas também pode não funcionar em outro por muitas razões.

Reafirmando sua crença, FRASCARI cita o Arquiteto francês Jean Labatut ${ }^{1}$ :

"Quaisquer que sejam os espaços aéreos, as superfícies e as dimensões envolvidas, o estudo preciso e a execução esmerada dos detalhes comprovam a grandeza da arquitetura. $\mathrm{O}$ detalhe conta a história." (LABATUT Apud FRASCARI, 2006, P 540)

Não se trata de uma novidade pensar que o detalhe exerce um papel capaz de modificar o sentido da obra arquitetônica, sobretudo analisando que a construção em si muitas vezes segue padrões repetidamente, fazendo com que a obra procure sua identidade através dos detalhes.

\section{PRODUÇÃO BIBLIOGRÁFICA}

A carência de bibliografia específica muitas vezes faz com que, ao longo de sua carreira, o arquiteto ou profissional da área de desenho crie sua própria identidade gráfica, e junto dela diferentes formas de representação que traduzam o que deve ser construído. A partir deste contato com o desenho técnico, o profissional aprende então a desenvolver uma linguagem gráfica que traz consigo informações formadoras de um padrão próprio. Tal padrão se encarrega pela transmissão de ideias, porém, muitas vezes acaba se distanciando de uma concepção formal com padrão de desenho específico.

Considerando que "o desenho é uma poderosa ferramenta analítica para o próprio criador" (MATOSO e VALLADARES e, 2002, p. 02) a representação gráfica pode ser considerada um dos principais instrumentos de comunicação entre o profissional, sua criação e todos os personagens que fazem parte da execução de um projeto.

O mercado de livros técnicos dispõe de alguma bibliografia sobre detalhamento arquitetônico, o que é resultado da escassa produção na área, porém, tais iniciativas ainda não supriram a atual deficiência. A criação de padrões de detalhamento podem esclarecer ideias sobre o projeto e auxiliar na criação de soluções para resolver problemas funcionais e de design.

Em 1936, o arquiteto alemão Ernst Neufert publicou o livro "Architect's Data". O livro é considerado uma referência universal para arquitetos, engenheiros e estudantes como um manual de construção que reúne fundamentos, normas e prescrições sobre recintos, edifícios, exigências e dimensões tomando o ser humano como medida e objetivo. Desde sua publicação, já foi traduzido em 18 idiomas incluindo o português, onde recebeu o título de "A Arte de Projetar em Arquitetura" (Figura 1).

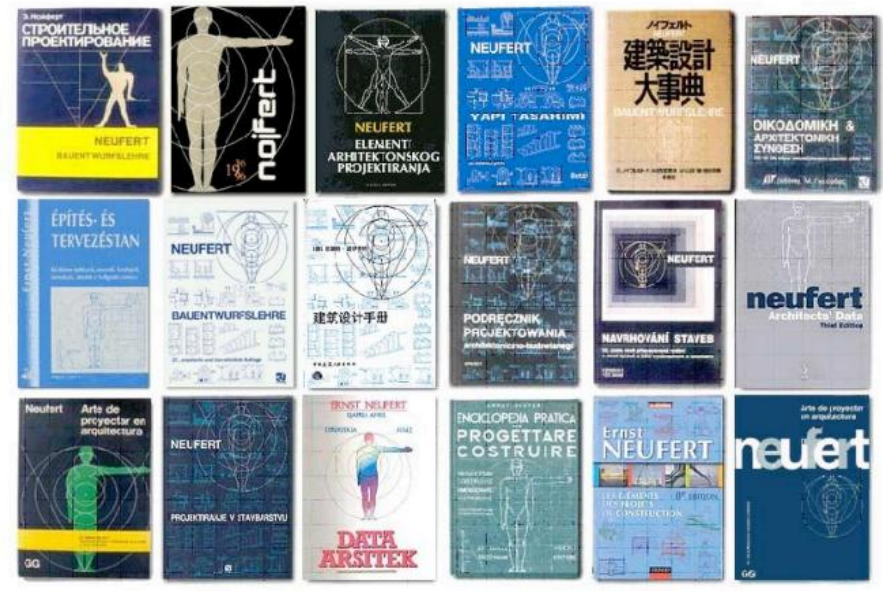

FIGURA 01: Algumas das principais edições do livro "A Arte de Projetar em Arquitetura".

SANTOS o considera como "o livro de arquitetura mais bem sucedido de todos os tempos.", e cita VOIGT para chamar a atenção em relação à linguagem de representação adotada (Figura 02):

"Neufert havia compreendido que a existência do cinema, da fotografia e da propaganda comercial modificara necessariamente os hábitos de leitura. Nessa "época ótica", segundo a expressão de Otto Neurat, o livro enquanto um meio de comunicação só teria sucesso caso se aproximasse do leitor com ajuda de meios visuais. Assim, a "Arte de Projetar em Arquitetura" era constituída, em grande parte, por pequenos desenhos, cujos traços já não são ilustrações do texto, mas sim, carregam uma necessária mensagem em si mesmos." (VOIGT Apud SANTOS, 2010, p.06)

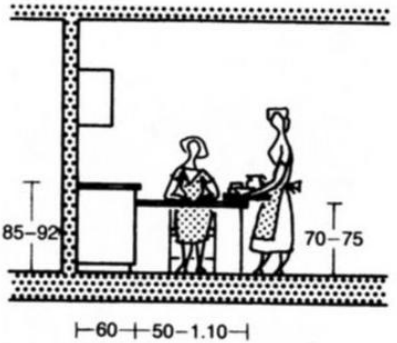

(15)
Pull-out/swivelling table

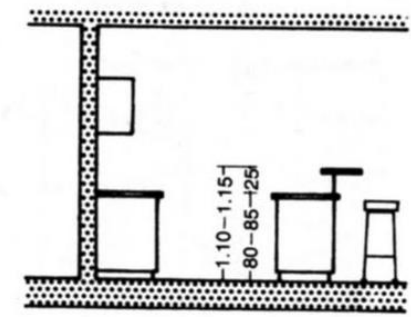

(16)
FIGURA 02: Exemplo de uma das primeiras bibliografias de referência para desenho técnico - NEUFERT, Ernst. A Arte de Projetar em Arquitetura. 
A ultima edição do livro traz atualizações e complementações, abordando temas como fachadas de madeira, hotéis, varandas cobertas, entre outros conteúdos. O livro ainda não dispõe de conteúdo digital reutilizável e não considera o detalhamento arquitetônico como foco principal, porém se tornou uma das principais referências em representação arquitetônica, fato que pode ser associado à falta de outras traduções.

Até os anos 70, poderia ser considerada a hipótese de que livros como A Arte de Projetar seriam o tipo de publicação que mais se relacionava com o tema detalhamento arquitetônico. Em 1977 foi lançado o livro The Professional Practice of Architectural Detailing (A Prática Profissional de Detalhamento Arquitetônico) trazendo consigo temas como CAD (Computer Aided Design) e apresenta os conceitos básicos de preparação de detalhes arquitetônicos através de mais de 600 ilustrações.

O livro teve sua $4^{\mathrm{a}}$ publicação em 2011 (Figura 03), quando recebeu o título de The Professional Practice of Architectural Working Drawings (A Prática Profissional de Desenhos de Trabalho Arquitetônico) onde aborda temas como a influência das tecnologias BIM através da representação de modelos tridimensionais, incluindo estudos de caso de projetos.

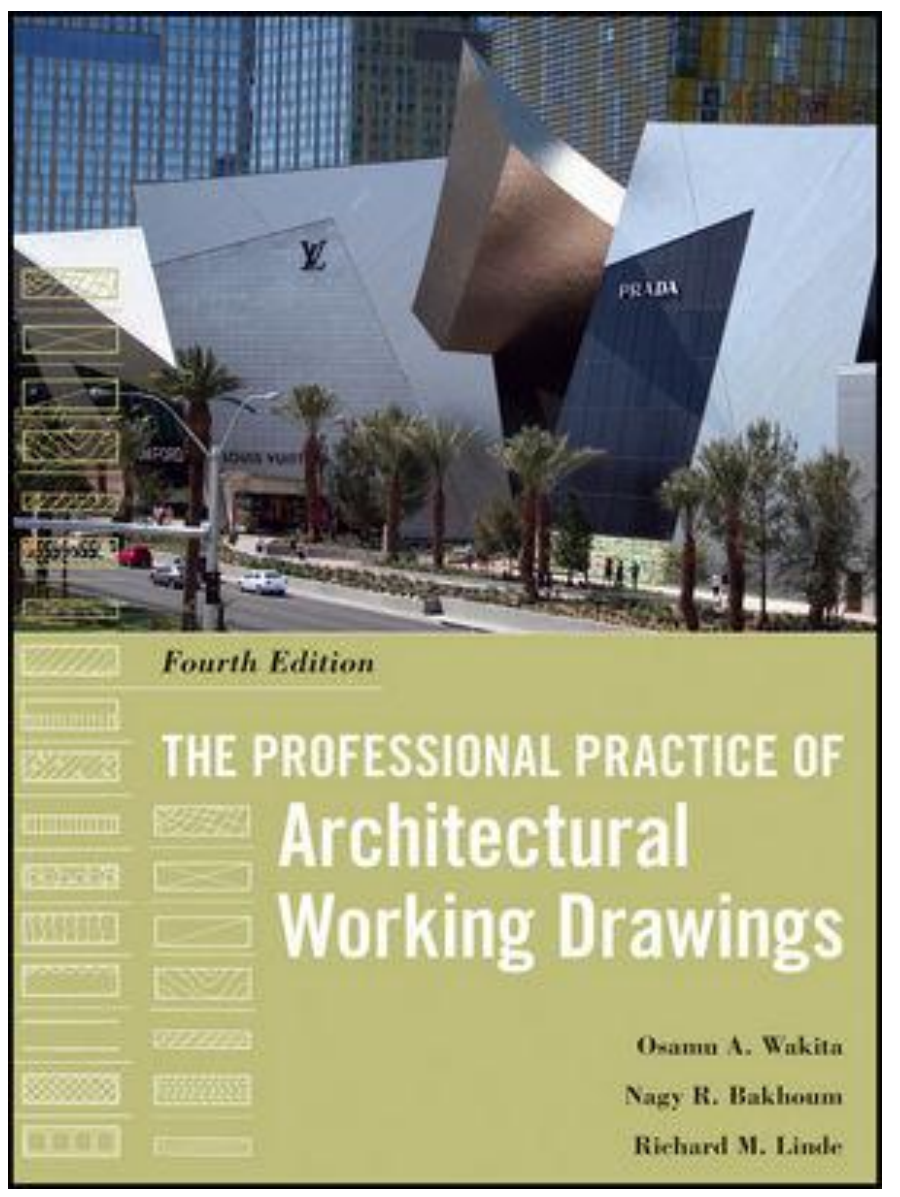

FIGURA 03: The Professional Practice of Architectural Working Drawings.

Considerando as últimas publicações na área de detalhamento arquitetônico, podem ser citados autores como Edward Allen e Patrick Rand com o livro Architectural Detail lançado em 2007, e mais recentemente Edward R. Ford, que lançou The Architectural Detail. Além destes, foram lançados entre 2007 e 2012 vários títulos pela autora Virginia McLeod que trata do tema detalhamento relacionando-o com outros temas como paisagismo, construções contemporâneas, construções em vidro, arquitetura doméstica, entre outros, além de disponibilizar junto do livro um CD com os modelos apresentados nos formatos EPS e DWG (FIGURA 04).
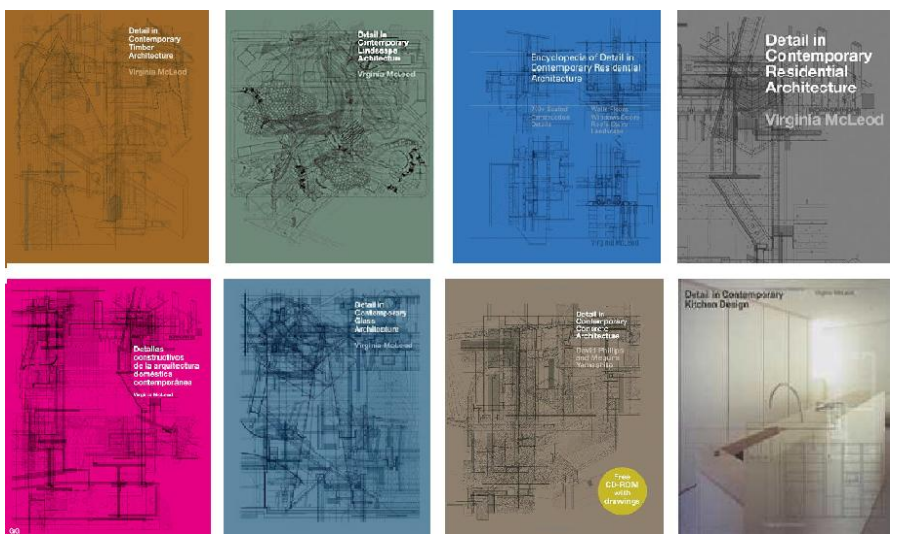

FIGURA 04: Algumas das principais publicações de Virginia McLeod sobre detalhamento relacionado a diferentes temas aliados à produção arquitetônica.

As publicações citadas acima vêm mostrando que a blibliografia sobre detalhes arquitetônicos já vem ocupando seu espaço no mercado e que este fato pode ser considerado uma consequência da relevância do tema, porém esta ainda não é uma realidade no Brasil. No que se refere à penetração destes livros no mercado brasileiro, apenas alguns títulos estão disponíveis; menor ainda é o número de traduções para a língua portuguesa.

\section{UTILIZAÇÃO DE FERRAMENTAS DIGITAIS COMO RECURSO DIDÁTICO E PROFISSIONAL}

Analisando algumas metodologias sugeridas por PENIDO e SOUZA, as quais eles chamam de pedagogias de projeto, que "consiste numa estratégia de produção de material didático a partir de um curso abordando o tema Detalhes Arquitetônicos", percebe-se que as experiências didáticas aplicadas nesta área pelo autor caminham ao encontro da digitalização do material didático que, sendo padronizado, pode inclusive servir como recurso para os 
profissionais que trabalham com desenho técnico como arquivos digitais de referência, tutoriais técnicos específicos e animações gráficas.

A utilização de ferramentas digitais como recurso didático e/ou profissional vem se tornando uma prática cada vez mais constante e vem conquistando seu espaço tanto nos setores educativos quanto profissionais. Mesmo tratando-se de um nicho ainda em ascensão, a utilização de mídias digitais e da tecnologia já vem se incorporando na educação através da necessidade de atualização e disseminação do conhecimento.

A criação de um Ambiente Virtual de Aprendizagem para publicar, armazenar e disponibilizar documentos permite organizar, selecionar e distribuir informações de acordo com as necessidades do usuário. Segundo LEINER, uma biblioteca digital é:

“(...) a coleção de serviços e de objetos de informação, com organização, estrutura e apresentação que suportam o relacionamento dos utilizadores com os objetos de informação, disponíveis direta ou indiretamente via meio eletrônico / digital." (LEINER, 1988, p.1)

Sendo assim, a transmissão de informações e conhecimento através da internet atua de maneira a eliminar barreiras físicas e a falta de acessibilidade causada pela distância.

No que diz respeito à produção didática de detalhamento arquitetônico, percebe-se que normalmente esta iniciativa parte de profissionais da área e de universidades. $\mathrm{Na}$ apresentação de seu livro, COSTA descreve sua justificativa ao decidir escrevê-lo:

"Durante todos os meus anos de vida como profissional na área de desenho, pude constatar a carência e a necessidade de uma bibliografia específica que atendesse as necessidades de aprendizagem e consulta por parte dos profissionais da área de desenho de Detalhamento de Arquitetura. Nossa preocupação fundamental foi suprir a deficiência de tais no mercado de livros técnicos." (COSTA, 1997, p. 5)

Em 2011, a FEEVALE criou o CDDC - um Catálogo Digital de Detalhamento da Construção. Trata-se de um website para uso dos alunos do curso de Arquitetura e Urbanismo da Universidade FEEVALE. No site encontram-se publicações e materiais produzidos pelos laboratórios do curso, focado no detalhamento da construção (Figura 05). O material explorou diferentes maneiras para elucidar e exemplificar detalhes e aspectos construtivos, disponibilizando textos, desenhos, fotos, modelos e outras mídias.

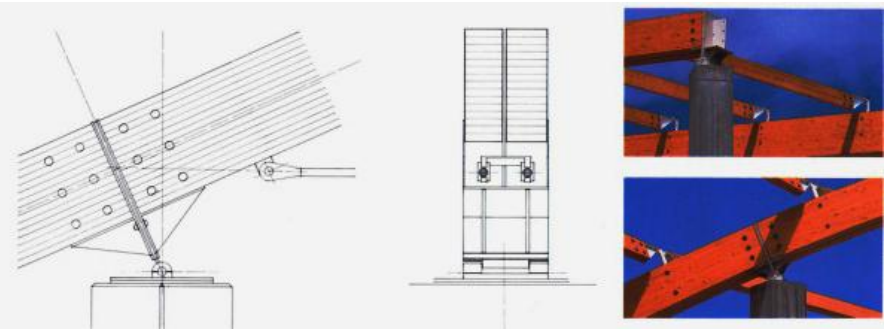

FIGURA 05: Detalhe da estrutura de cobertura do Bercy 2 Shopping Centre (projeto de Renzo Piano)

Quando se trata da produção de bibliotecas digitais para uso profissional, o mercado é ainda mais escasso. Percebe-se dois tipos principais de criação: a primeira parte de empresas que digitalizam seu catálogo de produtos, o que resulta na divulgação de seus equipamentos e na otimização do trabalho dos profissionais que através deste podem ter acesso aos desenhos técnicos digitalizados. Entre as coleções mais conhecidas atualmente aparece o DecaCAD, que funciona como um gerenciador que insere blocos dos produtos vendidos pela marca Deca disponiveis em formato .dwg compatíveis com o software AutoCAD. Este aplicativo por ser instalado no software através de download e está disponível no site da empresa.

Recentemente a empresa desenvolveu uma biblioteca BIM, sendo a primeira fabricante nacional a criar um aplicativo para projetos com esse conceito. O DecaBIM (Figuta 06) é compatível com o software Revit, e contou com o apoio de uma equipe multidisciplinar no seu processo de criação e desenvolvimento como profissionais da área de arquitetura e construção e a própria empresa fabricante do aplicativo.

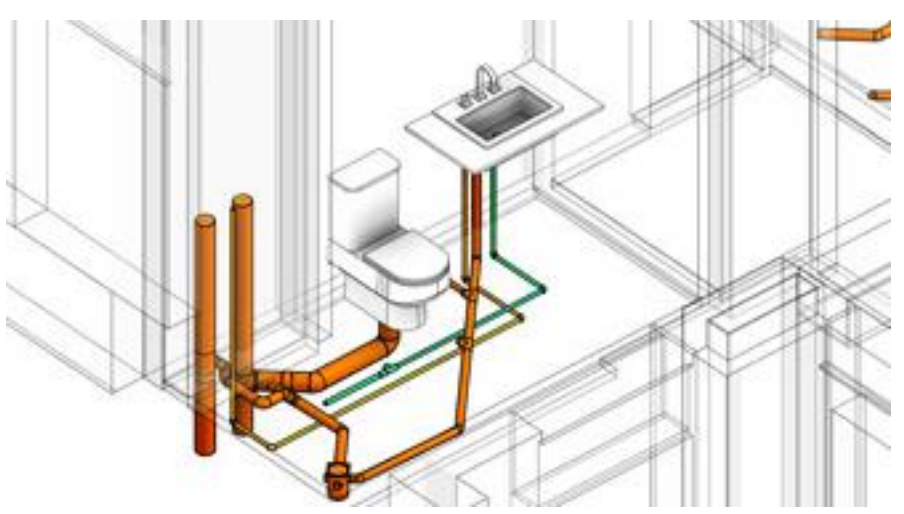

FIGURA 06: Imagen do Plugin DecaBIM.

Em uma das suas mais recentes atualizações, o software Sketchup adicionou novos componentes à sua biblioteca. Trata-se de componentes BIM (Building Information Modeling) flexíveis nos quais pode-se adicionar atributos, relações e comportamentos que se configuram conforme as alterações feitas no desenho através da 
adição de metadados. Alguns dos componentes foram criados por usuários do Sketchup, mas em maioria são criados por fabricantes e revendedores como uma forma de promover seus produtos.

Através da inserção de componentes BIM no Sketchup, outros plugins foram surgindo com esta mesma plataforma. Com incentivo público, o estúdio brasileiro Superfície.org junto de um grupo de arquitetos e programadores desenvolveu uma ferramenta que possibilita o orçamento de projetos criados nesta plataforma: o Bim Bon. Uma das principais funcionalidades do Bim Bon é uma ferramenta de busca de objeto, que funciona como o atual Armazén 3D da Google . A diferença é que os objetos deste plugin vêm com diversas informações associadas como fabricante, modelo, preço e outros.

O Bim Bon cadastra componentes e materiais e usa a informação dos objetos e das áreas do projeto para a montagem de orçamentos, diferenciando pisos, paredes, acabamentos e detalhamentos. A ideia destaque do plugin é a possibilidade da criação de orçamentos, porém através de sua biblioteca pode-se obter componentes dinâmicos de todas as marcas e fabricantes cadastrados. Entre eles destacam-se a Deca, Suvinil, Eliane, Docol, Mosarte, entre outros, que somam mais de 300 fabricantes de materiais de construção e decoração, com catálogo de produtos que possibilitam baixar modelos $3 \mathrm{D}$ e consultar informações técnicas de artigos para cozinha e banheiro, portas, janelas, piso, parede, estrutura, telhado, etc (Figura 07).

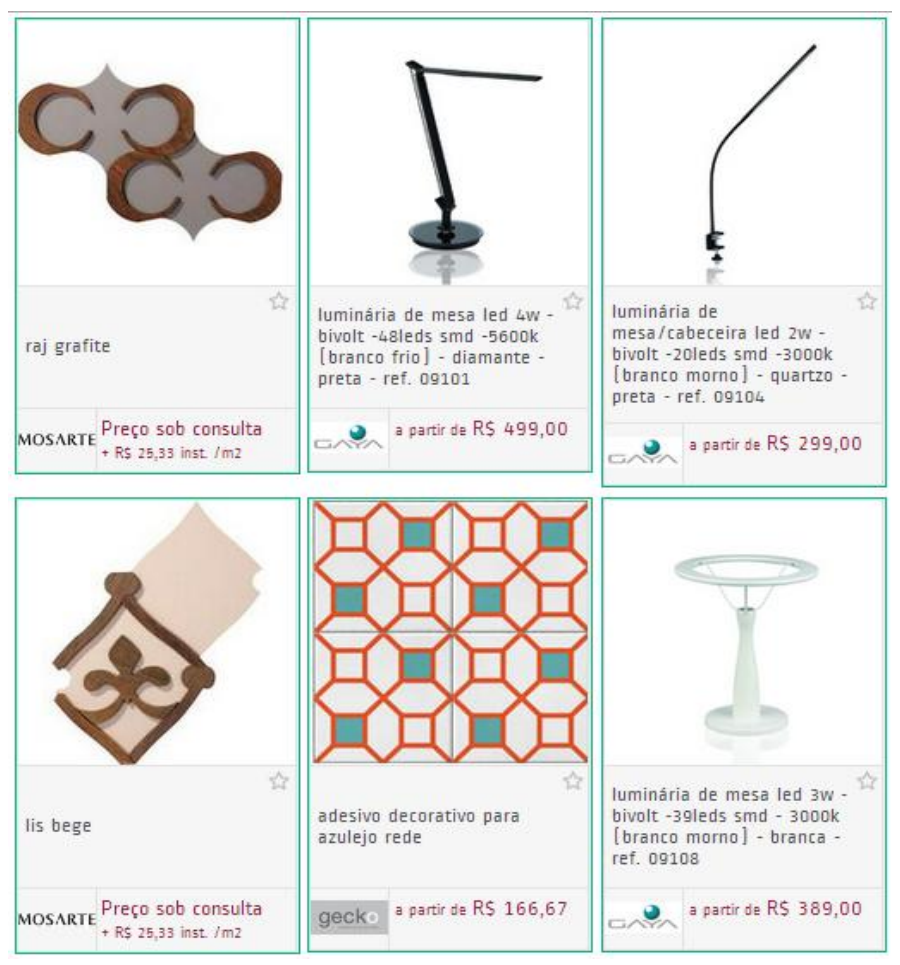

FIGURA 07: Catálogo de Produtos BimBon.
Mais recentemente, foram lançadas outras ferramentas BIM para o Sketchup, e junto delas aplicativos que compatibilizam e preparam os modelos paramétricos para impressão 3D (i.materialize), corte a laser (slice) e o $S T L$ for SketchUp.

A utilização de tecnologias digitais como a parametrização e a prototipagem possibilitam a materialização de detalhes e do projeto arquitetônico em si. A possibilidade de materializar detalhes permite maior compreensão por parte de todos os agentes do projeto, tanto a nível acadêmico quanto profissional, incluindo o próprio arquiteto.

"O emprego de novas tecnologias no processo de projeto permitiu aos arquitetos ultrapassar os limites da complexidade, alcançando com muito mais eficiência as necessidades e exigências do ser humano." (PUPO e CELANI, 2009, p.11)

Como o uso de equipamentos de fabricação digital se baseia nos conceito "file to factory", em que arquivos digitais 2D ou 3D são enviados diretamente para equipamentos de controle numérico, faz ainda mais sentido diponibilizar esses arquivos em bibliotecas na Internet.

\section{Conclusão}

A partir da análise de todos os dados apresentados neste artigo, mostra-se necessário e possível o desenvolvimento de bibliotecas digitais de detalhamento arquitetônico, sobretudo no que se refere à criação de modelos em 3D. Percebe-se o grande avanço que a plataforma BIM possibilitou a inovação nesta área. Considerando que hoje existem bibliotecas digitais que fornecem modelos de fabricantes de uma série de produtos, torna-se possível o desenvolvimento de bibliotecas onde possam ser encontrados componentes de detalhamento arquitetônico para representação gráfica que visem interoperabilidade entre softwares, aprendizagem e materialização de modelos.

Assim como as bibliografias existentes, o a padronização através da criação de bibliotecas digitais - tanto $2 \mathrm{D}$ quanto 3D - podem servir como uma solução geral para um problema recorrente enfrentado por arquitetos e profissionais da área que é a carência de um padrão que norteie o desenvolvimento de projetos e desenhos, o que faz com que cada escritório ou profissional crie seu próprio padrão de representação gráfica ao longo da carreira. Ao estabelecer um vocabulário comum de desenho pode facilitar a comunicação, documentação e aprendizado, torna-se possível a reutilização de desenhos e soluções de projeto. 
A utilização de ferramentas digitais como recurso didático apresenta-se como uma alternativa viável dentro do processo de projeto. Impulsionar esta prática no ambiente acadêmico pode auxiliar na modernização da formação dos alunos além de usufruir de todas as possibilidades de aprendizagem alcançadas através da parametrização, prototipagem e materialização de modelos (detalhes) como uma nova prática de ensino do projeto arquitetônico.

\section{Referências}

Livros

ALLEN, Edward. RAND, Patrick. Architectural Detailing: Function - Constructibility - Aesthetics. John Wiley \& Sons: New Jersey, 2012. Parcialmente disponível em http://www.wiley.com/WileyCDA/WileyTitle/productCd-111842896X,descCdgoogle_preview.html

BIZLEY, Graham. Architecture in Detail II. Routledge: United Kingdom, 2010.

COSTA, Antonio Ferreira da. Detalhando a arquitetura. Rio de Janeiro: Zoomgraf-K, 1997. v.v. 2. 167 p. Ilus.

FORD, Edward R. The Architectural Detail. Princeton Architectural Press: New York, 2011.

MCLEOD, Virginia. Detail in Contemporany Residential Architecture. Laurence King Publishing: London, 2012.

NEUFERT, Ernst. Arte de Projetar em Arquitetura. 18 ed. São Paulo: Gustavo Gili, 2013.

REAS, Casey. MCWILLIAMS, Chandler. LUST. Form and Code in Design, Art and Architecture. Princeton Architectural Press: New York, 2010.

NEUFERT, Ernst. Arte de Projetar em Arquitetura. 18ª ed. São Paulo: Gustavo Gili, 2013.

Artigos e Apostilas

LEINER, Barry M. Metrics and digital libraries. D.Lib Magazine, 1988. Disponível em: http://www.dlib.org/dlib/july98/07editorial.html.

MATOSO, Danilo. VALLADARES, Porfírio. PROJETO DE INTERIORES: Apostila de projeto executivo e detalhamento. Escola de Arquitetura da UFMG - Departamento de Projetos. Disponível em: http://daniloarquiteto.files.wordpress.com/2008/11/apostila_exec_det.pdf

PENIDO, André R. SOUZA, Renato César F. Detalhes Arquitetônicos - Uma Experiência Didática Segundo A Pedagogia Dos Projetos Para A Produção De Material Didático. CONBEGE: Blumenau, 2011. Disponível em: http://www.abenge.org.br/CobengeAnteriores/2011/sessoestec/art1933.pdf Acessado em 28/09/2013.

Internet

DECA PARA PROFISSIONAIS: DecaCAD e DecaBIM. Disponível em: http://www.deca.com.br/deca-para-profissionais/decabim/ Acessado em: 29/09/2013.

SKETCHUP: Os componentes dinâmicos no SketchUp Pro 8. Disponível em: http://www.sketchup.com/intl/ptBR/product/dcspro.html Acessado em: 30/09/2013.

Catálogo Digital de Detalhamento da Construção - Curso de Arquitetura e Urbanismo da Universidade Feevale. Disponível em: http://cddcarqfeevale.wordpress.com/

Acessado em: 23/09/2013.

BIM BON - Produtos e Fabricantes. Disponível em: http://www.bimbon.com.br/produtos Acessado em: 23/09/2013.

BIM BON - Aprimoramentos da Versão 0.6 - http://arquiteturacomsketchup.blogspot.com.br/. Acessado em: 01/10/2013. 